\title{
Tuberculous Otitis Media-A Rare Entity or a Missed Diagnosis
}

\author{
D. S. Deenadayal*, Bashetty Naveen Kumar, Vyshanavi Bommakanti, K. Lakshmi Sameeri \\ H.O.D Dept of ENT and HNS, Yashoda Hospital, Secunderabad, India \\ Email: "aarticlinic@yahoo.com
}

Received 4 December 2015; accepted 8 March 2016; published 11 March 2016

Copyright () 2016 by authors and Scientific Research Publishing Inc.

This work is licensed under the Creative Commons Attribution International License (CC BY). http://creativecommons.org/licenses/by/4.0/

(c) (i) Open Access

\begin{abstract}
Objective: The aim of the study is to look for indicators of Tuberculous Otitis Media in all cases of chronic suppurative otitis media. Study Design: This is a retrospective study. Setting: This study was conducted in a tertiary care centre. Subjects and Methods: Detected cases of tuberculous otitis media (ТВOM) cases studied retrospectively from January 2011 to December 2013 were included in the study. Investigations for tuberculosis were considered in the cases showing suspicious pale granulation tissue in the external auditory canal and middle ear and also in cases showing exuberant pale granulation tissue during mastoid surgery where Zeihl Nielson staining, histopathology, molecular genetic study was done. Cases showing positive result were included in the study. Results: During the study period 751 cases of CSOM were seen in outpatient room of which 18 cases of TBOM were diagnosed. Three patients were diagnosed preoperatively and 181 underwent surgery of which 15 cases were diagnosed positive, from the tissue obtained during the procedure. Direct smear was positive in four cases, concentration techniques in seven cases. Line probe assay was taken as diagnostic in all the cases. None of the cases were positive on histopathology. Conclusion: Tuberculous otitis media is often missed as the classical features are not seen in all cases of ТВОМ. The absence of these should not stop the clinician from diagnosing the disease. Suspicious tissue should therefore be tested properly to avoid missing the diagnosis and to prevent any complications.
\end{abstract}

\section{Keywords}

Tuberculous Otitis Media, Molecular Genetic Assay Line Probe Assay, Anti Tuberculous Therapy

\section{Introduction}

Tuberculosis is a chronic granulomatous disease, caused by Mycobacterium tuberculosis-characterized by

${ }^{*}$ Corresponding author.

How to cite this paper: Deenadayal, D.S., Kumar, B.N., Bommakanti, V. and Sameeri, K.L. (2016) Tuberculous Otitis MediaA Rare Entity or a Missed Diagnosis. International Journal of Otolaryngology and Head \& Neck Surgery, 5, 65-72.

http://dx.doi.org/10.4236/ijohns.2016.52011 
formation of caseating granulomas.

Resurgence of this disease noted in 1990s [1] is being attributed to multi drug resistance, increase in population living in poor socioeconomic conditions (overcrowding \& poor nutrition) and increase in the incidence of HIV.

Tuberculous affecting the middle ear is very rare [2]. It accounts for less than $1 \%$ of cases of chronic suppurative otitis media [3].

There are various reasons to explain why this entity goes unrecognized in many cases. Readily recognized manifestations of tuberculosis are well described and discussed, compared to rare ones. Low prevalence of tuberculous otitis media (TBOM), scarcity of literature and knowledge, varied clinical features from silent mastoiditis to intracranial complications have often resulted in disease being a "missed diagnosis". Over this, the classical triad of painless otorrhoea, multiple perforations and facial palsy —is not seen in many cases, misleading the clinician. The diagnostic utility of various tests in diagnosing tuberculosis is variable, making it even more difficult to identify cases with tuberculosis.

Late recognition or missing a diagnosis means prolonged illness, disability, irreversible complications, unnecessary medical and surgical therapies and their costs to the patient, despite the availability of effective therapies. In children, hearing impairment may lead to long-term effects on language, auditory and cognitive development, hindering their education prospects.

Complications occurring due to tuberculous otitis media are very severe and life threatening as compared to pyogenic otits media warranting early diagnosis and treatment [4].

Efficacy of medical management with anti-tuberculous therapy (ATT) is well proven with good results in these cases [5].

Role of surgery is still controversial. We have been diagnosing cases by examination of tissue obtained during mastoid exploration/reconstructive procedures done for chronic otitis media.

This study is conducted to emphasize the features of the disease that help in early recognition, and also, on the efficacy of combined surgical and medical management in treating the disease.

\section{Methods}

A retrospective study was done and patients who were diagnosed as having tuberculous otitis media as per line probe assay were included in the study.

Approval from the Institute's Scientific Committee has been taken for conducting this study.

The study was conducted from January 2011 to December 2013.

A total of 751 cases of Chronic Suppurative Otitis Media (CSOM) were seen in the outpatient department of which 18 cases were found to be positive. Three cases were diagnosed preoperatively in whom there was pale granulations-in the external canal and middle ear. A total of 181 patients underwent surgery for CSOM. During surgery there was strong indication of tuberculosis in 28 patients, samples were obtained and tested with the standard protocol (Figure 1). Those found positive were included in the study. Out of these 28 patients-15 were found to be positive with LPA (Line probe assay) which was the diagnostic tool in our study.

In all patients detailed history of the patients was taken, including past and family history of tuberculosis. Pure tone Audiometry was done, a mean of pure tone average at 500, 1000 and $2000 \mathrm{~Hz}$ was taken to know the degree of hearing loss. Radiological and microbiological studies were done.

Inclusion criteria:

1) Patients of chronic ear disease not responding to culture based antibiotic therapy

2) Suspected cases showing pale granulation tissue in the external auditory canal or middle ear

3) Patients having multiple perforations

4) Patients posted for tympanoplasty or mastoid exploration with the presence of pale granulations/polypoid tissue

This tissue obtained was first tested for presence of acid fast bacilli with Ziehl Nielsen staining. Specimens with low or no yield were again tested after employing concentration techniques. During histopathological examination, presence of caseation necrosis, multinucleated giant cells and lymphatic infiltration are carefully observed.

A Sample of the tissue was also sent for Molecular Genetic study with line probe Assay (MTBDR plus assay) (Figure 2) for identification of Mycobacterium tuberculosis and resistance to Isoniazid and Rifampicin. 


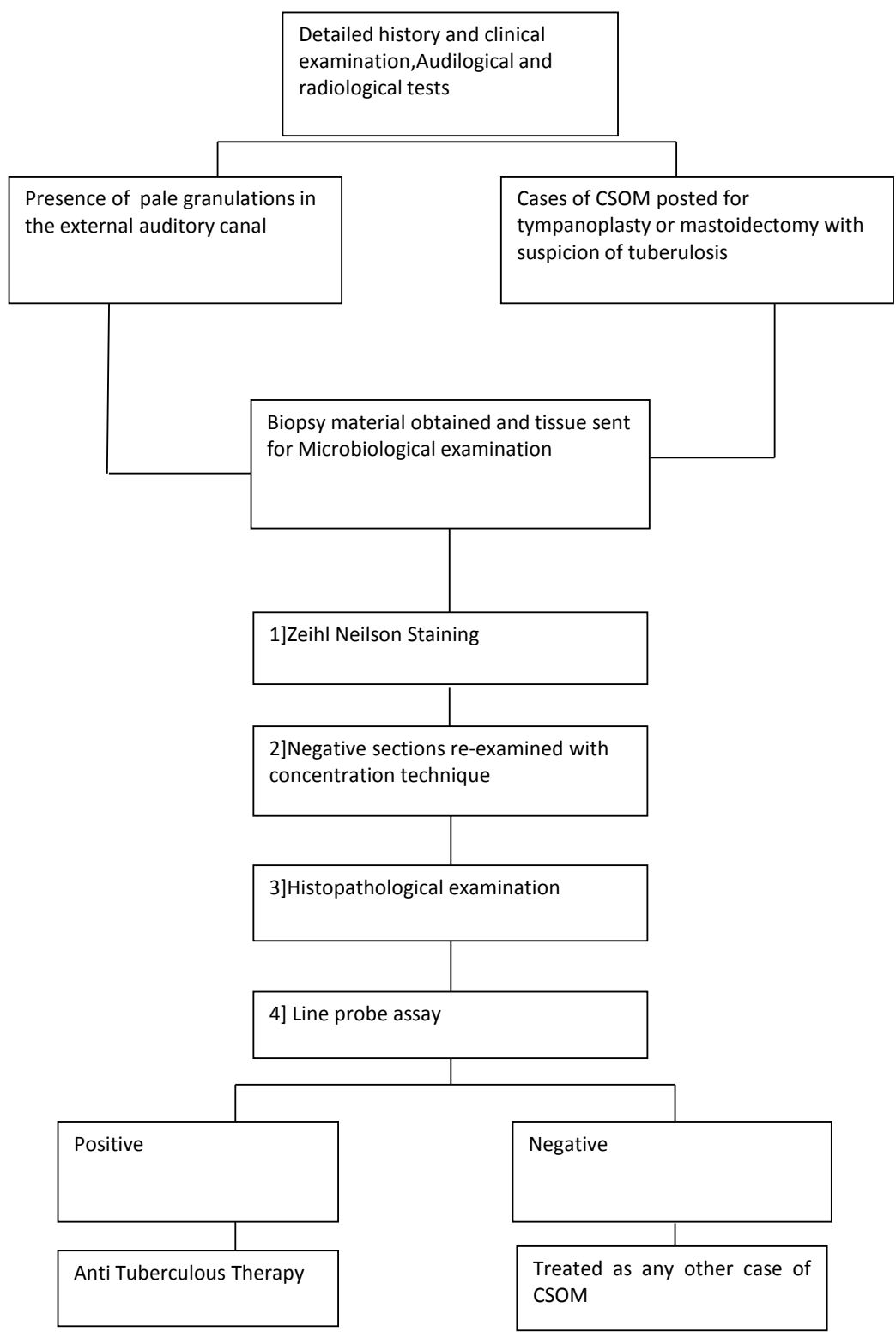

Figure 1. Protocol followed for all cases.

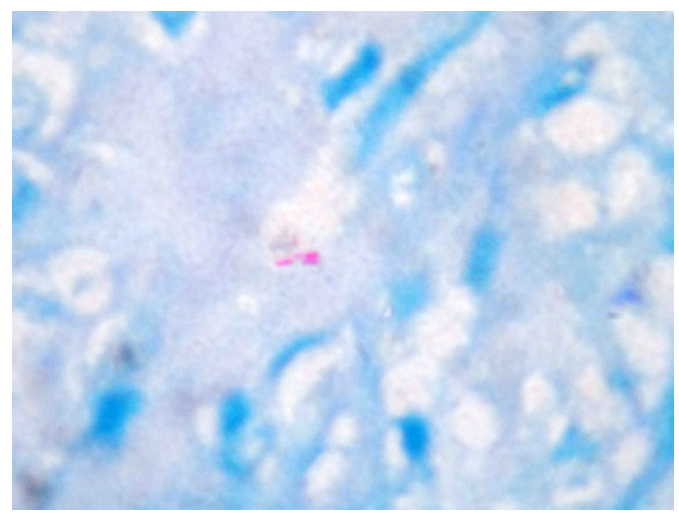

Figure 2. Line probe assay showing tuberculous bacilli. 
This is based on DNA-Strip technology. Here, presence of bands in Conjugate control zone (CC), and Amplification Control zone and TUB zone are verified, for presence of $M$. tuberculosis. Bands in three locus control zones-rpoB, kat $G$ and Inh $A$ help in identifying Rifampicin, high level and low level Isoniazid resistance respectively.

A major strength of the line probe assay is that it is commercially available in a kit form and produces results in a day. Limitations include relatively high level of skill is required to run the assay (e.g. DNA processing), costs, and inability of the assay to accommodate new mutations [6].

Patients with positive result were started on anti tubercular therapy. The first was two months intensive phase where they were prescribed a combination of Isoniazid, Rifampicin, Pyrazinamide and Ethambutol three times a week. This is followed by a 4-month regimen of isoniazid and Rifampicin three times a week. Defaulters and failures were treated with three months intensive phase of three times a week-combination of Isoniazid, Rifampicin, Pyrazinamide, Ethambutol and Streptomycin for two months; and Isoniazid, Rifampicin, Pyrazinamide and Ethambutol for one month. During continuation phase, Isoniazid, Rifampicin, and Ethambutol were administered for five months.

Patients who had negative results were treated as any other case of chronic otitis media.

\section{Results}

During the study period 751 cases of CSOM were seen in outpatient room of which 18 cases of TBOM were diagnosed. Three patients were diagnosed preoperatively and 181 underwent surgery of which 15 cases were diagnosed positive, from the tissue obtained during the procedure. 8 were males and 10 were females. Age range of the patients was between 17 and 61 years, with maximum number of patients (33\%) in their thirties. The Second peak was observed in the 6th decade (26.6\%).

Among the operated cases six were revision cases (40\%) and all of them were operated earlier for the same complaints. The duration of presentation ranged from three months to 38 years. None of the patients had pulmonary tuberculosis either in the past or at the time of presentation ( $0 \%)$. In no case was the family history positive.

The presenting complaints in the order of frequency are listed in Table 1 and the Examination findings are listed in Table 2.

Imaging of temporal bones showed sclerotic mastoids in twelve cases. The granulation tissue obtained during surgery was tested for tuberculosis and it was positive in these cases. The most common site of appearance of granulation tissue was the mastoid antrum (Figure 3), followed by the mastoid cavity proper and the middle ear. Incus was the most common ossicle $(n=9,60 \%)$ to be eroded, followed by malleus $(n=8,53.3 \%)$ and stapes suprastructure $(n=2,13.3 \%)$. Dehiscence of facial canal was seen in three of the cases $(20 \%)$.

\section{Table 1. Presenting complaints.}

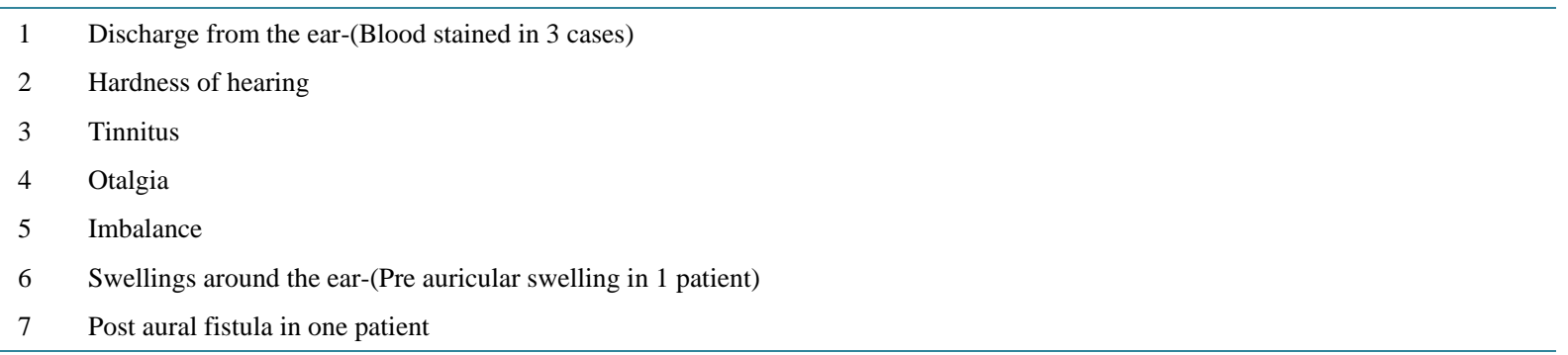

\section{Table 2. Examination findings.}

\footnotetext{
1 Edema of external auditory canal with granulations (3 cases) (Figure 4)

2 Multiple perforation (1 case)

3 Central perforation (8 cases) with granulations in the middle ear

$4 \quad$ Thick and dull intact tympanic membrane (2 cases) (Figure 5)

5 Perforation of tympanic membrane with discharge (4 cases)
} 


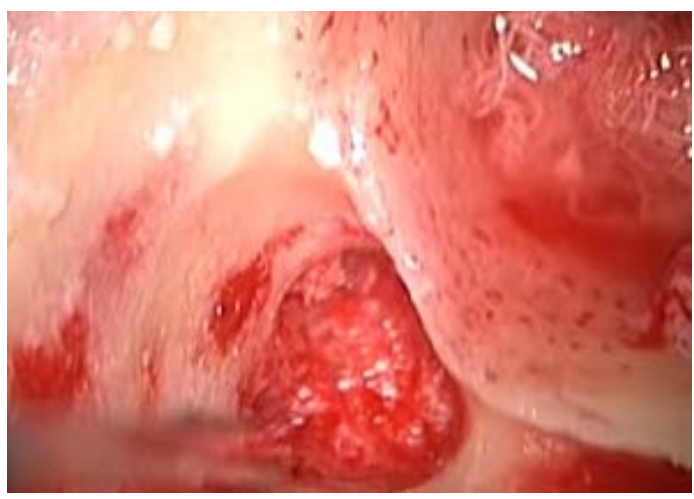

Figure 3. Pale granulations in the mastoid antrum.

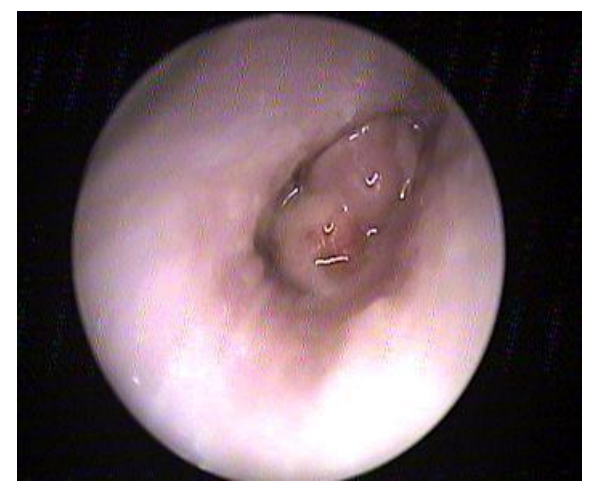

Figure 4. Pale granulations in the external auditory canal.

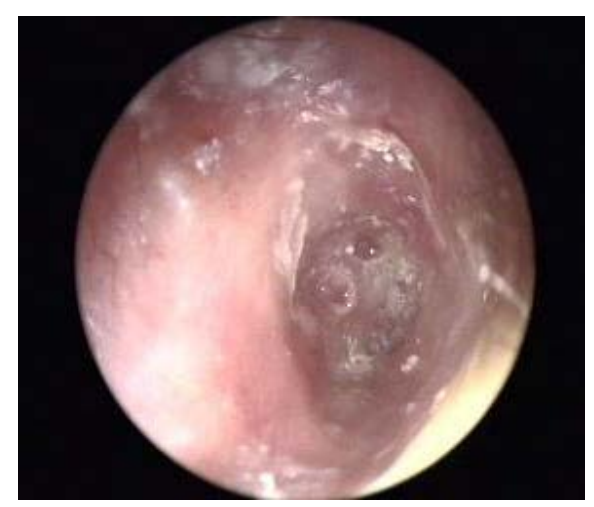

Figure 5. Thick and dull tympanic membrane.

Direct smear was positive in four cases (22.2\%), concentration techniques in seven cases (38.8\%). Line probe assay was the diagnostic tool in all the cases. None of the cases were positive on histopathology. There was no evidene of caseous necrosis or epitheloid cells ( $0 \%)$.

Anti-tuberculous therapy was started within 7 - 10 days of surgery, except in one case where it was started after 15 days. There were two defaulters. One patient had no further complaints after resuming ATT. In another case the ear started discharging after two weeks of stopping medication at 2.5 months, and showed no response even after reinstatement of ATT. That patient developed a small central perforation in the anteroinferior quadrant.

All the other cases (88.8\%) responded well to ATT, with normal healing of the post-aural wound and proper uptake of the graft (Figure 6). All the mastoid cavities showed good epithelialization.

The mean PTA also improved from 53.73 to $27.1 \mathrm{~dB}$. 


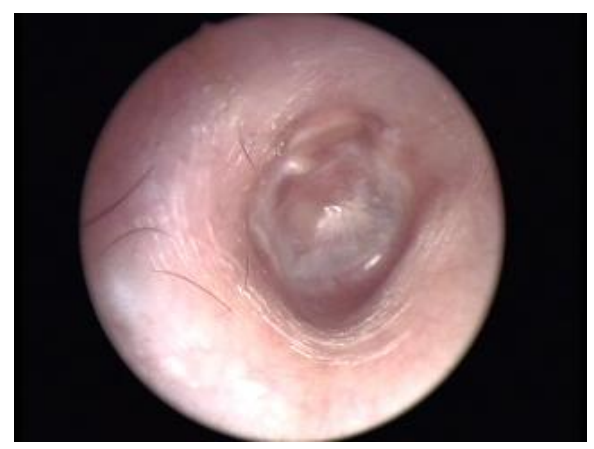

Figure 6. Post operative picture showing good uptake of the graft with healthy ear.

Patients were followed up for a period of one year. All patients responded well (88.8\%) with no complications and had healthy ears.

\section{Discussion}

We conducted the study on patients who came with a complaint of chronic discharging ear not responding to medical therapy. They were investigated with audiometry, examination of discharge, and radiological studies of the temporal bones.

In our outpatient room we examined 751 patients with CSOM of which 18 cases were diagnosed as tuberculous otitis media, hence the incidence of TBOM in our study was $0.02 \%$. A study done by Weiner GM report incidence of tuberculous otitis media to be $0.04 \%$ of cases of chronic otitis media [7] which is much lower as compared to our study. A total of 181 cases were operated for chronic suppurative otitis media of which 15 cases were diagnosed as tuberculous otitis media. Hence TBOM accounted for $8.2 \%$ of the operated cases of chronic suuppurative otitis media

Males are usually more affected than females with a ratio of 1.4:1 as studied by Nishiike $\mathrm{S}$ et al. but in our study the ratio was 0.8:1 [8]. As supported by Cho et al. the highest incidence of the disease in patients is around their thirtees. This correlated with our study [9].

The most common complaint was chronic ear discharge and hardness of hearing which is also seen in various other studies [10].

In our study Multiple perforations could be seen in only one case (5.55\%) which correlates with a clinical study done in twelve patients of tuberculous otitis media. NONE of the patients in that study had multiple perforation [8].

Intra Operative findings showed pale granulation tissue as the constant finding. The characteristic bony sequestration and cheesy material as described by Abes, was not found in any of our cases [11]. Ossicular necrosis was seen involving the incus, malleus, followed by stapes. Erosion of fallopian canal was seen in 3 cases.

In our study we did not encounter any complications, which contradicts a study done by Jesic $\mathrm{S}$ who reported that otogenic complications were more in tuberculous otitis media as compared to cholesteatoma [12]. In our series, detection rate of Mycobacteria by molecular genetic methods was about 4.5 times higher compared to direct staining and 2.5 times higher compared to concentration techniques.

Ziehl-Nielsen staining should not be considered as a sensitive test, since tuberculous lesions of the ear show low bacterial concentrations [13]. Obtaining a positive microbiologial culture is also difficult as the bacterial count is low and other microorganisms (staphylococcus, pseudomonas, Klebsiella) interfere with the growth [13].

There were no histologically positive cases in our study, but a study by windle taylor reports 20/22 cases to be histologically positive and histology was considered as a diagnostic tool-which contradicts our study [14]. In our study we used molecular genetic studies to facilitate detection of bacteria in low counts, and they have the advantage of labelling drug resistance. But Mycobacterium-specific PCR and interferon-gamma release assay cannot distinguish between active, inactive or post-infective conditions [15].

A study done by Kriukov et al. could diagnose tuberculous process, bacteriologically in 9\% cytological in $27.3 \%$, pathomorphological in $18 \%$ and PCR diagnostics in $55 \%$. While in our study cytological in $22.2 \%$ 
Table 3. Key points.

Key points

1) Tuberculous otitis media is a rare entity-Is it Rare? Or is the diagnosis missed?

2) Classical clinical features of tuberculosis are rarely seen and atypical findings which help in diagnosing should be kept in mind.

3) Zn staining for tuberculosis should not be considered as a sensitive test.

4) Histopathology is considered as diagnostic in the diagnosis of tuberculosis but in case of tuberculous otitis media it is difficult to appreciate the classical features as the bacterial count is low in the specimen due to prolonged use of topical antibiotics.

5) Molecular genetic studies like line probe assay not only helps in the diagnosis of tuberculosis but even identifies the sensitivity to drugs isoniazid and rifampcin.

6) Tests for diagnosing tuberculosis should be repeated even if its initially negative whenever there is a strong clinical suspicion.

7) Surgery is required for tissue sampling and for clearance of the disease.

pathologically in 0 percent and with PCR diagnostics (line probe assay) in all the cases. Hence PCR-Diagnositics increase the sensitivity of diagnosis of tuberculosis [16].

Line probe assay has a specificity of $96 \%-100 \%$ and sensitivity of $94 \%-100 \%$, thus it can accurately diagnose tuberculosis [7].

Diagnostic efficacy is variable for all the tests, hence the diagnosis of tuberculosis still remains a challenge for otolaryngologists. Hence whenever there is strong clinical suspicion investigating the patient with various tests is imperative.

The mean PTA was improved from 53.73 to $27.1 \mathrm{~dB}$. Thus surgery helped patients in achieving a healthy middle ear cavity with a good hearing outcome.

In our study, we found that with proper ATT, healing rates are similar to any non-TBOM cases. There was no formation of post aural fistula or graft rejection. As observed by Aremu [17] in his study, our patients also complained of wet feeling of the ear (rather than discharge) in the initial post operative period-for three months. A mean follow up for one year was done and all patients were doing well except for the defaulters.

\section{Conclusions}

Tuberculous otitis media is often missed as the classical triad of painless otorrhoea, multiple perforations and facial palsy are not seen in all cases of TBOM. The absence of these should not stop the clinician from diagnosing the disease.

Suspicious tissue should therefore be tested properly-to avoid missing the diagnosis and to prevent any complications. The key points are summarized in Table 3.

\section{References}

[1] Karande, S. and Bavdekar, S.B. (2002) Children and Multidrug-Resistant Tuberculosis in Mumbai (Bombay), India. Emerging Infectious Diseases, 8, 1360-1361. http://dx.doi.org/10.3201/eid0811.020513

[2] Varty, S., Vaidya, D., Parasram, K., Prabhat, D. and Joshi, S. (2000) Tuberculous Otitis Media—Are We Missing It? Indian Journal of Otolaryngology and Head \& Neck Surgery, 52, 143-146.

[3] Moya, P.A., Malinvaud, D., Mimoun, M., Huart, J. and Bonfils, P. (2008) Tuberculous Otomastoiditis: Advantage of MRI in the Treatment Survey. Revue de Laryngologie Otologie Rhinologie (Bord), 129, 301-304. (In French) http://www.ncbi.nlm.nih.gov/pubmed/19408515

[4] Prakash, M. and Johnny, J.C. (2015) Intra Cranial Complications of Tuberculous Otitis Media. J Pharm Bioallied Sci., 7, 51. http://dx.doi.org/10.4103/0975-7406.155795

[5] Kwon, M., Choi, S.H. and Chung, J.W. (2010) Roles of an Anti-Tuberculosis Medication and Surgery in Patients with Tuberculous Otitis Media. Acta Oto-Laryngologica, 130, 679-686. http://dx.doi.org/10.3109/00016480903311260

[6] Catanzaro, A., Rodwell, T.C., Catanzaro, D.G., et al. (2015) Performance Comparison of Three Rapid Tests for the Diagnosis of Drug-Resistant Tuberculosis. PLoS One, 10, e0136861. http://dx.doi.org/10.1371/journal.pone.0136861

[7] Weiner, G.M., O’Connell, J.E. and Pahor, A.L. (1997) The Role of Surgery in Tuberculous Mastoiditis: Appropriate Chemotherapy Is Not Always Enough. Journal of Laryngology and Otology, 111, 752-753.

http://www.ncbi.nlm.nih.gov/pubmed/9327015 
[8] Nishiike, S., Irifune, M., Doi, K., Osaki, Y. and Kiuchi, N. (2003) Tuberculous Otitis Media: Clinical Aspects of 12 Cases. Annals of Otology, Rhinology \& Laryngology, 112, 935-938. http://www.ncbi.nlm.nih.gov/pubmed/14653361 http://dx.doi.org/10.1177/000348940311201104

[9] Cho, Y.-S., Lee, H.-S., Kim, S.-W., et al. (2006) Tuberculous Otitis Media: A Clinical and Radiologic Analysis of 52 Patients. The Laryngoscope, 116, 921-927. http://dx.doi.org/10.1097/01.mlg.0000214861.87933.00

[10] Vaamonde, P., Castro, C., Garciasoto, N., Labella, T. and Lozano, A. (2004) Tuberculous Otitis Media: A Significant Diagnostic Challenge. Otolaryngology_-Head and Neck Surgery, 130, 759-766. http://dx.doi.org/10.1016/j.otohns.2003.12.021

[11] Abes, G.T., Abes, F.L.L.B. and Jamir, J.C. (2011) The Variable Clinical Presentation of Tuberculosis Otitis Media and the Importance of Early Detection. Otology \& Neurotology, 32, 539-543. http://dx.doi.org/10.1097/MAO.0b013e3182117782

[12] Jesić, S., Stosić, S., Milenković, B., et al. (2009) Middle Ear Tuberculosis: Diagnostic Criteria. Srpski Arhiv za Celokupno Lekarstvo, 137, 346-350. http://www.ncbi.nlm.nih.gov/pubmed/19764586

[13] Lee, P.Y. and Drysdale, A.J. (1993) Tuberculous Otitis Media: A Difficult Diagnosis. The Journal of Laryngology \& Otology, 107, 339-341. http://www.ncbi.nlm.nih.gov/pubmed/8320523 http://dx.doi.org/10.1017/S0022215100122972

[14] Windle-Taylor, P.C. and Bailey, C.M. (1980) Tuberculous Otitis Media: A Series of 22 Patients. The Laryngoscope, 90, 1039-1044. http://www.ncbi.nlm.nih.gov/pubmed/6770207

[15] Liktor, B., Liktor, B., Kálmán, J., Horváth, B., Sziklai, I. and Karosi, T. (2014) Primary Tuberculosis of the Middle Ear Cleft: Diagnostic and Therapeutic Considerations. European Archives of Oto-Rhino-Laryngology, 271, 2083-2089. http://dx.doi.org/10.1007/s00405-014-2977-7

[16] Kriukov, A.I., Garov, E.V., Ivořlov, A.Y.U., Shadrin, G.B., Sidorina, N.G. and Lavrova, A.S. (2015) The Clinical Manifestations and Diagnostics of Otitis Media Caused by Tuberculosis. Vestnik Otorinolaringologii, 80, 28-34. (In Russian) http://www.ncbi.nlm.nih.gov/pubmed/26288206

[17] Aremu, S.K. and Alabi, B.S. (2010) Tuberculous Otitis Media: A Case Presentation and Review of the Literature. BMJ Case Reports, 2010, bcr0220102721. 